\title{
TI subgroups and depth 3 subgroups in simple Suzuki groups
}

\author{
Hayder Abbas Janabi, László Héthelyi and Erzsébet Horváth* \\ Communicated by Michael Giudici
}

\begin{abstract}
In this paper, we determine the TI subgroups of the simple Suzuki groups $\operatorname{Sz}(q)$. More generally, we determine those nontrivial subgroups that are disjoint from some of their conjugates. It turns out that the latter are exactly those subgroups that have ordinary depth 3. The Sylow 2-subgroups of simple Suzuki groups belong to the class of so-called Suzuki 2-groups, which have been studied extensively by Higman. These results were extended later by Goldschmidt, Shaw, Shult, Gross, Wilkens and Bryukhanova. As a corollary of our investigations, we get some interesting results for the Sylow 2-subgroups of Suzuki groups, as well. We relate this to an open problem on Suzuki 2-groups, and we ask a question concerning that. We also give some characterization of Suzuki groups.
\end{abstract}

\section{Motivation and results}

In an earlier paper [14], we determined the ordinary and combinatorial depth of several subgroups of the simple Suzuki groups $\mathrm{Sz}(q)$. For the definitions of these notions, see Definition 2.5 and Definition 2.8 in Section 2. There are several ways to define ordinary depth; a good summary of these can be found in [18]; see also [6, Definition 3.5] and the introduction of [8]. The notion of combinatorial depth was introduced in [2, Definition 3.2]. We use [2, Theorem 3.9] as an equivalent definition. A subgroup $L \leq G$ is called $T I$ if, for every $x \in G$, from $L^{x} \cap L \neq\{1\}$, it follows that $x \in N_{G}(L)$. In an arbitrary group, non-normal TI subgroups are always of combinatorial depth three, hence also of ordinary depth three. Moreover, nontrivial subgroups having a disjoint conjugate are always of ordinary depth three in every group. However, in general, the converse is not true, e.g. $L=A_{5}$ has in $G=A_{7}$ ordinary depth 3 , but there is no element $x \in G$ such that $L \cap L^{x}=\{1\}$. (A similar example is $A_{6}$ in $A_{10}$.)

The first author was supported by the Stipendium Hungaricum PhD fellowship at the Budapest University of Technology and Economics. The second and the third author were supported by the NKFI Grants No. 115288 and 115799. 
It is an open problem how to characterize subgroups of ordinary depth 3 in a group-theoretical way in an arbitrary group.

We will show in Theorem 1.3 that, in the case of simple Suzuki groups, subgroups of ordinary depth 3 are exactly those nontrivial subgroups that have a disjoint conjugate.

In Theorem 1.1, we also characterize those nontrivial TI subgroups of $\operatorname{Sz}(q)$ which are non-cyclic elementary abelian 2-subgroups. These are exactly those subgroups that are conjugate to the center of a Sylow 2-subgroup of a smaller Suzuki subgroup $\mathrm{Sz}(s) \leq \mathrm{Sz}(q)$. This property can help the recognition of those subgroups of $\operatorname{Sz}(q)$ which are isomorphic to a Suzuki subgroup $\operatorname{Sz}(s) \leq \operatorname{Sz}(q)$. Recognition of Suzuki groups in $\operatorname{GL}(4, q)$ is considered in some recent papers; see $[1,4]$. In another paper [23], Suzuki groups were used to construct some block designs. So results about intersections in Suzuki groups might also be helpful in combinatorial investigations.

In this paper, we will use the following notation. Let $G$ be the simple Suzuki group $\operatorname{Sz}(q)$, where $q=2^{2 m+1}$. Let $F$ be a fixed Sylow 2-subgroup of $G$. Then $N_{G}(F)=F H$, where $|H|=q-1$. Let $A_{1}$ and $A_{2}$ be Hall subgroups of $G$ of orders $q+2 r+1$ and $q-2 r+1$, respectively, where $r=2^{m}$; see Theorem 2.1 and also [17, Theorem 3.10, Chapter XI] and [22, Theorem 4.12].

We will denote by $K_{2^{n}}$ an elementary abelian subgroup of order $2^{n}$. The center $Z(F)$ of the Sylow 2-subgroup $F$ of the Suzuki group $\operatorname{Sz}(q)$ will be of order $q=2^{2 m+1}$, and we will denote $2 m+1$ by $f$. We also suppose that $m>0$. The main results of this paper are the following theorems.

Theorem 1.1. If $G=\mathrm{Sz}(q)$ is a simple Suzuki group, then $G$ has the following TI subgroups.

(i) Cyclic subgroups of prime order and the trivial subgroup.

(ii) Subgroups $F, H, A_{1}, A_{2}$, their characteristic subgroups and the conjugates of these.

(iii) An elementary abelian subgroup $K_{2^{n}}$ of order $2^{n}>2$ is a TI-subgroup if and only if it is the center of a Sylow 2-subgroup of a simple Suzuki subgroup $G_{1} \leq G$, or a conjugate to it. This holds if and only if $n>1$ and $n \mid f$. (Remember $|Z(F)|=2^{f}$.) These subgroups are exactly those non-cyclic elementary abelian 2-subgroups of $G$ that have combinatorial depth 3 .

All other nontrivial subgroups are not TI.

Remark 1.2. This classification of TI subgroups shows that [1, Theorem 2.1 (7)] is wrong; the centralizer of an element of order 4 cannot be TI. 
Theorem 1.3. Subgroups of ordinary depth 3 of a simple Suzuki group $G=\operatorname{Sz}(q)$ are the following.

(i) Every nontrivial subgroup contained in a maximal subgroup different from a conjugate of $N_{G}(F)$.

(ii) $F$ and all its nontrivial subgroups, and the conjugates of these.

(iii) All nontrivial subgroups $U=F_{1} K$ of $N_{G}(F)$, where $F_{1}<F$ and $K \leq H$, and the conjugates of these subgroups.

Moreover, a nontrivial subgroup $L \leq G=\mathrm{Sz}(q)$ is of ordinary depth 3 if and only if there exists an element $x \in G$ with $L \cap L^{x}=\{1\}$.

As a corollary, we get a characterization of Suzuki groups.

Corollary 1.4. Let $G$ be a simple Zassenhaus group acting on $q+1$ points, where $q$ is a 2-power. Then the following are equivalent.

(i) $G \simeq \operatorname{Sz}(q)$.

(ii) If $F \in \operatorname{Syl}_{2}(G)$, then every subgroup not containing a conjugate of the subgroup $F$ has a disjoint conjugate.

Proof. By Theorem 1.3 and by the description of subgroups of $\mathrm{Sz}(q)$ in Theorem 2.1, we have that (i) implies (ii). For the other direction, observe that the only simple Zassenhaus groups with $q$ even are $\operatorname{Sz}(q)$ and $\operatorname{PSL}(2, q)$. In $\operatorname{PSL}(2, q)$, there are dihedral subgroups of order $2(q+1)$, that are not disjoint from their conjugates; see [7].

We also have a wider class of groups where ordinary depth 3 is equivalent to having a disjoint conjugate.

Corollary 1.5. Let $G$ be a simple Zassenhaus group acting on $q+1$ points, where $q$ is an odd power of 2. Then the following are equivalent for a nontrivial subgroup $L \leq G$.

(i) The subgroup $L \leq G$ has ordinary depth 3 .

(ii) The subgroup $L \leq G$ has a disjoint conjugate.

Proof. Since these Zassenhaus groups are $\operatorname{Sz}(q)$ and $\operatorname{PSL}(2, q)$, we have to prove the equivalence for these groups. In Theorem 1.3, we proved the equivalence for $\operatorname{Sz}(q)$. In [7], this is proved for $\operatorname{PSL}\left(2,2^{2 n+1}\right)$.

We also get some information about Sylow 2-subgroups of Suzuki groups. 
Corollary 1.6. Every Sylow 2-subgroup of $G=\operatorname{Sz}(q)$ is the union of conjugates of the Sylow 2-subgroups of a smallest simple Suzuki subgroup $G_{1}$ contained in $G$. If $F \in \operatorname{Syl}_{2}(G)$ and $F_{1} \in \operatorname{Syl}_{2}\left(G_{1}\right)$ are contained in $F$, then $F=\bigcup_{x \in N_{G}(F)} F_{1}{ }^{x}$. Every element of order 4 of $F$ is in exactly one conjugate of $F_{1}$, and any two of the conjugates of $F_{1}$ in this union either have trivial intersection or their intersection is their center, which is a conjugate of $\Omega_{1}\left(F_{1}\right)$.

We give a proof of this in Section 3.

Remark 1.7. It is easy to see that $F=\bigcup_{x \in N_{G}(F)} F_{2}^{x}$, where $F_{2} \simeq C_{4}$, and in this union, any two conjugates of $F_{2}$ either have trivial intersection, or the intersection is a conjugate of $\Omega_{1}\left(F_{2}\right)$. This is because there are two conjugacy classes of order 4 elements in $G$. Let they be $K_{G}(a)$ and $K_{G}\left(a^{-1}\right)$, where $a \in F$. Consider $\bigcup_{x \in N_{G}(F)}\langle a\rangle^{x}$. This will contain all elements of order 4 in $F$, i.e. all elements of $F \backslash Z(F)$. However, each involution in $F$ is a square of an element of order 4, since this is true for at least 2 involutions and the other involutions are conjugate by elements of $H \leq N_{G}(F)$. Hence we are done.

About the structure of the paper: In Section 2, we give some properties of the Suzuki groups that are needed in the proofs; we also give the definitions of combinatorial and ordinary depth of a subgroup. In Section 3, we give the proof of Theorem 1.1 and of Corollary 1.6. In Section 4, we prove Theorem 1.3. At the end of the paper, we formulate two questions.

\section{Preliminaries}

Suzuki groups $\operatorname{Sz}(q)$ are twisted groups of Lie type ${ }^{2} B_{2}(q)$, where $q:=2^{2 m+1}$. If $m>0$, then they are simple. Suzuki groups are also doubly transitive permutation groups on $q^{2}+1$ points; they belong to the class of Zassenhaus groups. Suzuki groups also can be defined as subgroups of $\operatorname{GL}(4, q)$. The order of $\operatorname{Sz}(q)$ is $\left(q^{2}+1\right)(q-1) q^{2}$. The order of $\mathrm{Sz}(q)$ is not divisible by 3 ; however, it is always divisible by 5 . For further information, see [14].

We will use the following results on the Suzuki groups; see [17, Theorem 3.10, Chapter XI] and [22, Theorem 4.12].

Theorem 2.1 (Suzuki). Let $G=\mathrm{Sz}(q)$, where $q=2^{2 m+1}$ for some positive integer $m$. Then $G$ has the following subgroups.

(1) The Hall subgroup $N_{G}(F)=F H$, a Frobenius group of order $q^{2}(q-1)$, where $F \in \operatorname{Syl}_{2}(G)$ and $H$ is cyclic of order $q-1$.

(2) The dihedral group $B_{0}=N_{G}(H)$ of order $2(q-1)$. 
(3) The cyclic Hall subgroups $A_{1}, A_{2}$ of orders $q+2 r+1, q-2 r+1$, respectively, where $r=2^{m}$ and $\left|A_{1}\right|\left|A_{2}\right|=q^{2}+1$.

(4) The Frobenius subgroups $B_{1}=N_{G}\left(A_{1}\right)$ and $B_{2}=N_{G}\left(A_{2}\right)$ of orders $4\left|A_{1}\right|$ and $4\left|A_{2}\right|$, respectively.

(5) Subgroups of the form $\mathrm{Sz}(s)$, where $s$ is an odd power of 2, $s \geq 8$, and $q=s^{n}$ for some positive integer $n$. Moreover, for every odd 2-power $s$, where $s^{n}=q$ for some positive integer $n$, there exists a subgroup isomorphic to $\mathrm{Sz}(s)$.

(6) Subgroups (and the conjugates of the subgroups) of the above groups.

Remark 2.2. The group ${ }^{2} B_{2}(2):=\mathrm{Sz}(2)$ is not simple; it is a Frobenius group of order 20. It is a subgroup of each simple $\operatorname{Sz}(q)$. Since 5 divides either $\left|A_{1}\right|$ or $\left|A_{2}\right|$, the group $\mathrm{Sz}(2)$ is a subgroup of $B_{1}$ or $B_{2}$ up to isomorphism.

Remark 2.3. By [25, Theorem 10], if $\mathrm{Sz}(s) \leq \mathrm{Sz}(q)$, then every subgroup of $\mathrm{Sz}(q)$ isomorphic to $\mathrm{Sz}(s)$ is also conjugate to it in $\mathrm{Sz}(q)$. Later in the paper, we will call the subgroups $\mathrm{Sz}(s) \leq \mathrm{Sz}(q)$ Suzuki subgroups of $\mathrm{Sz}(q)$. In the case of matrix generators of $\operatorname{Sz}(q)$ in $\operatorname{GL}(4, q)$, it is the subgroup generated by the same matrices when the matrix entries are restricted to $G F(s)$.

Theorem 2.4. Let $q=2^{2 m+1}, m>0, r=2^{m}$ and $G=\operatorname{Sz}(q)$.

(a) Let $i \in\{1,2\}$, and let $u_{i} \in A_{i}, u_{i} \neq 1$. Then $C_{G}\left(u_{i}\right)=A_{i}$. If $B_{i}=N_{G}\left(A_{i}\right)$, then $B_{i}=\left\langle A_{i}, t_{i}\right\rangle$, where $t_{i}$ is an element of order 4 , and $u^{t_{i}}=u^{q}$ for all $u \in A_{i}$. Moreover, $N_{G}\left(A_{i}\right)$ is a Frobenius group with kernel $A_{i}$.

(b) Let $F, H, A_{1}, A_{2}$ be as in Theorem 2.1. Then the conjugates of $F, H, A_{1}, A_{2}$ form a partition of $G$. In particular, $F, H, A_{1}, A_{2}$, their conjugates and the conjugates of their characteristic subgroups are TI sets in $G$.

The Sylow 2-subgroup $F$ of $G$ is a Suzuki 2-group. This means that it is a nonabelian 2-group, having more than one involution, and having a solvable group of automorphisms which permutes the set of involutions of $F$ transitively. See [16, p. 299] for details.

The group $F$ is a class 2 group of order $q^{2}$ and exponent 4 . Moreover, its center $Z(F)=F^{\prime}=\Phi(F)$ is of order $q$. The involutions in $F$ together with the identity element constitute $Z(F)$, and $F$ does not contain any quaternion subgroups. A nontrivial element of $F$ is real in $G$ if and only if it is an involution. (An element of a group $G$ is called a real element in $G$ if it is conjugate in $G$ to its inverse; see [10, p. 303].)

The subgroup $H$ acts sharply 1-transitively on the involutions of $F$, and on the nontrivial elements of $F / Z(F)$. The centralizer in $G$ of every nontrivial element 
of $F$ is a subgroup of $F$. For $m>0$, Suzuki groups are simple and their orders are congruent to $2 \bmod 3$.

Zassenhaus groups are doubly transitive permutation groups without any regular normal subgroup, where any non-identity element has at most two fixed points. Zassenhaus groups are always of degree $q+1$, where $q$ is a prime power. If $q$ is even, then there are two series of Zassenhaus groups PSL $\left(2,2^{n}\right)$ for $n>1$ and $\mathrm{Sz}\left(2^{2 m+1}\right)$ for $m>0$. If $q=p^{f}$ is odd and $f$ odd, then Zassenhaus groups are $\operatorname{PGL}\left(2, p^{f}\right)$ and $\operatorname{PSL}\left(2, p^{f}\right)$ if $p^{f} \neq 3$. If $f=2 m$, then beside $\operatorname{PGL}\left(2, p^{f}\right)$ and $\operatorname{PSL}\left(2, p^{f}\right)$, there exists a third type of Zassenhaus group $M\left(p^{f}\right)$. It can be constructed in the following way: let $H=\operatorname{PGL}\left(2, p^{f}\right)\langle\alpha\rangle \leq P \Gamma L\left(2, p^{f}\right)$, where $\alpha \in \operatorname{Aut}\left(G F\left(p^{f}\right)\right)$ of order 2 , raising elements of $G F\left(p^{f}\right)$ to the $m$-th power. There are 3 subgroups of $H$ of index 2 containing $\operatorname{PSL}\left(2, p^{f}\right): \operatorname{PGL}\left(2, p^{f}\right)$, $\operatorname{PSL}\left(2, p^{f}\right)\langle\alpha\rangle$ and $M\left(p^{f}\right)$. See [17, Chapter XI].

The notion of ordinary depth was originally defined for von-Neumann algebras; see [9]. Later, it was also defined for Hopf algebras; see [21]. For some recent results in this direction, see [12,13,19]. In [20] and later in [6], the depth of semisimple algebra inclusions was studied. The ordinary depth of a group inclusion $L \leq G$ (denoted by $d(L, G)$ ) is defined as the minimal depth of the group algebra inclusion $\mathbb{C} L \subseteq \mathbb{C} G$, studied in [2]. We will use an equivalent definition which is established in [6]. For the case of depth one and two, see $[3,20]$.

Let $G$ be a finite group, $L \leq G$ a subgroup. We introduce the ordinary depth as follows.

Two irreducible characters $\alpha, \beta \in \operatorname{Irr}(L)$ are related (denoted by $\alpha \sim_{G} \beta$ ) if they are constituents of the restriction $\left.\chi\right|_{L}$ for some $\chi \in \operatorname{Irr}(G)$. The distance $d_{G}(\alpha, \beta)=m$ is the smallest integer $m$ such that there is a chain of irreducible characters of $L$ such that $\alpha=\psi_{0} \sim_{G} \psi_{1} \sim_{G} \cdots \sim_{G} \psi_{m}=\beta$. If there is no such chain, then $d_{G}(\alpha, \beta)=-\infty$, and if $\alpha=\beta$, then the distance is zero. If $X$ is the set of irreducible constituents of $\left.\chi\right|_{L}$, then $m(\chi):=\max _{\alpha \in \operatorname{Irr}(L)} \min _{\psi \in X} d_{G}(\alpha, \psi)$.

Definition 2.5 ([6, Theorem 3.6, Theorem 3.10], [20, Corollary 3.2], [3, Theorem 1.7]). Let $L$ be a subgroup of a finite group $G$. The ordinary depth $d(L, G)$ is the minimal possible positive integer which can be determined from the following upper bounds.

(i) For $m \geq 1, d(L, G) \leq 2 m+1$ if and only if the distance between two irreducible characters of $L$ is at most $m$.

(ii) For $m \geq 2, d(L, G) \leq 2 m$ if and only if $m(\chi) \leq m-1$ for all $\chi \in \operatorname{Irr}(G)$.

(iii) $d(L, G) \leq 2$ if and only if $L$ is normal in $G, d(L, G)=1$ if and only if $G=L C_{G}(x)$ for all $x \in L$. 
Theorem 2.6 ([6, Theorem 6.9]). Suppose that $L$ is a subgroup of a finite group $G$ and $N=\operatorname{Core}_{G}(L)$ is the intersection of $m$ conjugates of $L$. Then $d(L, G) \leq 2 m$. If $N \leq Z(G)$ holds, then $d(L, G) \leq 2 m-1$.

Remark 2.7. It follows from the first part of Theorem 2.6 that if a non-normal subgroup $L \leq G$ has a disjoint conjugate, i.e. $L \cap L^{x}=\{1\}$ for some $x \in G$, then $d(L, G) \leq 4$. Since in this case $\operatorname{Core}_{G}(L)=\{1\}$, it follows from the second part of Theorem 2.6 that $d(L, G) \leq 3$. But since $L$ is not normal in $G, d(L, G)=3$. However, the converse is not true, e.g. $d\left(A_{5}, A_{7}\right)=3$, but $A_{5}$ does not have a disjoint conjugate in $A_{7}$.

The combinatorial depth can be defined as follows; see [2].

Definition 2.8. Let $L$ be a subgroup of the finite group $G$, and let $i \geq 1$. Then the combinatorial depth $d_{c}(L, G)$ of the subgroup $H$ in $G$ is defined in the following way.

(i) $d_{c}(L, G) \leq 2 i$ if and only if, for every $x_{1}, \ldots, x_{i} \in G$, there exist some $y_{1}, \ldots, y_{i-1} \in G$ with $L \cap L^{x_{1}} \cap \cdots \cap L^{x_{i}}=L \cap L^{y_{1}} \cap \cdots \cap L^{y_{i-1}}$.

(ii) Let $i>1$. Then $d_{c}(L, G) \leq 2 i-1$ if and only if, for every $x_{1}, \ldots, x_{i} \in G$, there exist some $y_{1}, \ldots, y_{i-1} \in G$ with

$$
L \cap L^{x_{1}} \cap \cdots \cap L^{x_{i}}=L \cap L^{y_{1}} \cap \cdots \cap L^{y_{i-1}}
$$

and $x_{1} h x_{1}^{-1}=y_{1} h y_{1}^{-1}$ for all $h \in L \cap L^{x_{1}} \cap \cdots \cap L^{x_{i}}$.

(iii) $d_{c}(L, G)=1$ if and only if, for every $x \in G$, there exists some $y \in L$ with $x h x^{-1}=y h y^{-1}$ for all $h \in L$. This holds if and only if $G=L C_{G}(L)$.

Remark 2.9. It is easy to see from Definition 2.8 that if a non-normal subgroup $L \leq G$ is TI, then $d_{c}(L, G)=3$. The converse is not true; we will see later that $C_{4}$ is not $\mathrm{TI}$ in $\operatorname{Sz}(q)$; however, $d_{c}\left(C_{4}, \operatorname{Sz}(q)\right)=3$ by [14, Theorem 4.1]. By Theorem [2, Theorem 4.1], we also know that $d(L, G) \leq d_{c}(L, G)$ for every subgroup $L \leq G$. For a non-normal subgroup $L \leq G$ to have a disjoint conjugate is a weaker property than being TI, e.g. in the case of simple Suzuki groups, every nontrivial 2 -subgroup has a disjoint conjugate; however, by Theorem 1.1, only some of them are TI.

\section{Proofs of Theorem 1.1 and of Corollary 1.6}

Proof of Theorem 1.1. We prove Theorem 1.1 in eight steps. It is obvious that every subgroup of prime order is TI. Now we examine the subgroups of maximal subgroups of $G$ to establish which are TI and which are not TI. 
(1) The subgroups $F, H, A_{1}, A_{2}$, their characteristic subgroups and their conjugates are TI. In particular, all subgroups of $H, A_{1}, A_{2}$ are TI; however, not every subgroup of $F$ is $T I$.

The first part follows from Theorem 2.4. Since $H, A_{1}, A_{2}$ are cyclic and TI, each subgroup of them is characteristic, hence TI. We will see below that not every subgroup of $F$ is TI.

(2) If $U \leq N_{G}(F)$ and $U=F_{1} H_{1}$, where $F_{1} \leq F, H_{1} \leq H$ are nontrivial subgroups, then $U$ is not $T I$.

Let $x \in N_{G}(H) \backslash F H$. Then $H_{1}^{x}=H_{1}$; hence $U^{x} \cap H \geq H_{1} \neq\{1\}$. Since $x \notin N_{G}(F)$, thus $F_{1}^{x} \not \subset F$ since $F$ is TI. Hence $U^{x} \cap U$ is a proper subgroup of $U$; thus $U$ is not TI.

(3) For every nontrivial subgroup $H_{1} \leq H$ and $u \in N_{G}(H) \backslash H$, the subgroup $\left\langle u, H_{1}\right\rangle$ is not $T I$.

Let $u \in N_{G}(H) \backslash H$; then this is an element of order 2 by Theorem 2.1 (2). So we may suppose that $u \in F_{1}$ for some subgroup $F_{1} \in \operatorname{Syl}_{2}(G)$. Since we know, see e.g. [22, Theorem 4.1 (b)], that all involutions in a Sylow 2-subgroup of $\operatorname{Sz}(q)$ are in the center of the Sylow 2-subgroup, thus we have that $u \in Z\left(F_{1}\right)$. Let $x \in F_{1} \backslash N_{G}(H)$. Then $\left(H_{1}\langle u\rangle\right)^{x} \cap H_{1}\langle u\rangle$ contains $u$. However, $x$ does not normalize $H_{1}$. Otherwise, it would also normalize $H$ since $H$ is TI. Hence $\left\langle u, H_{1}\right\rangle$ is not TI.

(4) If we take a nontrivial subgroup $\tilde{A}$ of $A_{i}$, and a nontrivial subgroup $C_{1} \leq C$ of a cyclic complement of order 4 in the Frobenius group $N_{G}\left(A_{i}\right)$, then $C_{1} \tilde{\tilde{A}}$ is not TI; in particular, $\mathrm{Sz}(2)$ is not TI.

Let $\tilde{A} \leq A_{i}, C \leq F_{1}$, where $F_{1} \in \operatorname{Syl}_{2}(G)$. Let

$$
n \in N_{F_{1}}(C) \backslash C=N_{F_{1}}(C) \backslash N_{G}\left(A_{i}\right)
$$

be an involution. Then $\left(C_{1} \tilde{A}\right)^{n} \cap C_{1} \tilde{A} \neq\{1\}$ since $C_{1}$ is characteristic in $C$. However, it does not contain $\tilde{A}$ since then $n \in N_{G}(\tilde{A}) \leq N_{G}\left(A_{i}\right)$, which is not the case. Hence $C_{1} \tilde{A}$ is not TI.

(5) Let $G_{1}:=\mathrm{Sz}(s)$ be a simple smaller Suzuki subgroup in $G$. The subgroups $U$ of $G_{1}$, whose order is divisible both by 2 and by some odd integer greater than 1 , are not TI.

Let $U_{2} \in \operatorname{Syl}_{2}(U)$, and let $U_{2} \leq P_{2} \in \operatorname{Syl}_{2}(G)$. Then $Z\left(P_{2}\right) \leq N_{G}\left(U_{2}\right)$; however, since $Z\left(P_{2}\right) \not \leq G_{1}$, we have that $Z\left(P_{2}\right) \not \leq U$. If $N_{P_{2}}\left(U_{2}\right) \leq N_{G}(U)$, then $U Z\left(P_{2}\right)$ is a subgroup of $G$. It cannot be a subgroup of any maximal subgroup of type $N_{G}\left(A_{i}\right), N_{G}(H)$ and $\mathrm{Sz}\left(s_{1}\right)$ since the orders of these subgroups are not divisible by $q$. If $U \leq N_{G}(S)$ for some $S \in \operatorname{Syl}_{2}(G)$, then $U$ is conjugate to a subgroup discussed in point (2); hence it is not TI. The subgroup $U Z\left(P_{2}\right)$ cannot 
be $G$ since $U$ is normal in it. So we can suppose that $N_{P_{2}}\left(U_{2}\right) \not \leq N_{G}(U)$. Let $x \in N_{P_{2}}\left(U_{2}\right) \backslash N_{G}(U)$. Then $U_{2} \leq U^{x} \cap U<U$; hence $U$ is not TI.

(6) Let $C:=\langle c\rangle$ be a cyclic subgroup of $F$ of order 4 . This is not TI.

By the proof of [17, Lemma 5.9, Chapter XI], we have that $C_{F}(C)=Z(F) C$ is of order $2 q$. Since $c$ is not real in $F$, we have that $N_{F}(C)=C_{F}(C)$. Thus there is an element $x \in F \backslash N_{F}(C)$. Let $u:=c^{2}$. Then $C_{G}(u)=F$, and hence $C^{x} \cap C$ contains $u$. Hence $C$ is not TI.

(7) Let us denote an elementary abelian subgroup of order $2^{n}$ in $F$ by $K_{2^{n}}$. The main results in this step are (e) and (f). This is exactly the content of (iii) in Theorem 1.1, which we will prove with the help of statements (a)-(d).

(a) Let $K_{2^{n}}=Z\left(S_{1}\right)$, where $S_{1} \in \operatorname{Syl}_{2}\left(G_{1}\right)$ and $G_{1}$ is a simple Suzuki subgroup of $G$. Then $K_{2^{n}}$ is TI in $G$.

We may assume that $S_{1} \leq F$. Suppose that $Z\left(S_{1}\right)^{x} \cap Z\left(S_{1}\right) \neq\{1\}$ for some $x \in G$. Then there exist involutions $a, b \in Z\left(S_{1}\right)$ with $a^{x}=b$. Since $F$ is TI, thus $x \in N_{G}(F)=F H$. Since $F$ acts trivially on $Z\left(S_{1}\right)$, we may suppose that $x \in H$. Moreover, $H$ acts sharply 1-transitively on the involutions of $F$. We also have that $N_{G_{1}}\left(S_{1}\right) \leq N_{G}(F)=F H$ since $F$ is TI. For some complement $H_{1}$ in $N_{G_{1}}\left(S_{1}\right), H_{1} \leq H$. However, $H_{1}$ also acts sharply 1 -transitively on the involutions of $Z\left(S_{1}\right)$. Thus $x \in H_{1} \leq N_{G_{1}}\left(S_{1}\right) \leq N_{G_{1}}\left(Z\left(S_{1}\right)\right)$. Hence $Z\left(S_{1}\right)$ is TI in $G$.

(b) If a non-cyclic elementary abelian subgroup of $G$ is TI, and its order is equal to $\left|Z\left(S_{1}\right)\right|$ for a Sylow subgroup $S_{1} \in \operatorname{Syl}_{2}\left(G_{1}\right)$ for some simple Suzuki subgroup $G_{1}$ of $G$, then this elementary abelian subgroup is the center of a Sylow 2 -subgroup of a subgroup conjugate to a simple Suzuki subgroup of $G$.

Let us suppose that a non-cyclic elementary abelian subgroup $K_{2^{n}} \leq F$ of order $2^{n}$ in $G$ is a TI set. We suppose that $2^{n}=\left|Z\left(S_{1}\right)\right|$ for $S_{1} \in \operatorname{Syl}_{2}\left(G_{1}\right)$, where $G_{1}$ is a simple Suzuki subgroup of $G$. The involutions of $K_{2^{n}}$ and their $H$-conjugates form blocks in $Z(F)$ since $K_{2^{n}}$ is TI in $G$. Since $H$ acts sharply 1-transitively on the involutions of $Z(F)$, hence $Z(F)$ is the disjoint union of different $H$-conjugates of $K_{2^{n}}$. We claim that the normalizers in $H$ of the elementary abelian TI subgroups $\tilde{K}_{2^{n}}$ of order $2^{n}$ of $G$ contained in $Z(F)$ are the same. To see this, note that no element of $H$ fixes any element in $F$; hence the elements of the cyclic subgroup $N_{G}\left(\tilde{K}_{2^{n}}\right) \cap H$ move the nonunit elements of $\tilde{K}_{2^{n}}$ sharply 1transitively; hence $N_{G}\left(\tilde{K}_{2^{n}}\right) \cap H$ has order $2^{n}-1$. Since $H$ is cyclic, it has only 1 subgroup of this order; thus each elementary abelian TI-subgroup of order $2^{n}$ in $Z(F)$ has the same normalizer in $H$. We know that $\tilde{K}_{2^{n}} \subseteq \bigcup_{h \in H} K_{2^{n}}^{h}$. We claim that $\tilde{K}_{2^{n}}$ is one of the conjugates of $K_{2^{n}}$. Suppose that $a \in \tilde{K}_{2^{n}} \cap K_{2^{n}}^{h_{1}}$ and $b \in \tilde{K}_{2^{n}} \cap K_{2^{n}}^{h_{2}}$ are two different involutions and $h_{1}, h_{2} \in H$. Then there exists a unique $h \in H \cap N_{G}\left(\tilde{K}_{2^{n}}\right)=H \cap N_{G}\left(K_{2^{n}}^{h_{1}}\right)$ with $a^{h}=b \in K_{2^{n}}^{h_{1} h} \cap K_{2^{n}}^{h_{2}}$. 
However, then $K_{2^{n}}^{h_{1}}=\left(K_{2^{n}}^{h_{1}}\right)^{h}=K_{2^{n}}^{h_{2}}$. Hence $\tilde{K}_{2^{n}}=K_{2^{n}}^{h_{1}}$. Thus all elementary abelian TI subgroups of order $2^{n}$ in $F$ are conjugate to $Z\left(S_{1}\right)$ for a Sylow subgroup $S_{1} \in \operatorname{Syl}_{2}\left(G_{1}\right)$ for some Suzuki subgroup $G_{1} \leq G$.

(c) Let $K_{2^{r}} \leq F$ be an elementary abelian subgroup of order $2^{r}$ in $G$. Then it is TI if and only if $N_{H}\left(K_{2^{r}}\right)=H_{1}$ is of order $2^{r}-1$.

Let $K_{2^{r}} \leq F$ be an elementary abelian subgroup of order $2^{r}$ with the property that $N_{H}\left(K_{2^{r}}\right)=H_{1}$ and $\left|H_{1}\right|=2^{r}-1$. Then $H_{1}$ permutes the elements of $K_{2^{r}} \backslash\{1\}$ sharply 1-transitively, and every $h \in H \backslash H_{1}$ transports each involution of $K_{2^{r}}$ outside this group. Thus $K_{2^{r}}$ is TI.

Conversely, let $K_{2^{r}} \leq F$ be TI. Since by [22, Theorem 4.1 (e), (f)] $H$ acts sharply 1-transitively on the involutions of $F$, if $a \in K_{2^{r}} \backslash\{1\}$, then for every $b \in K_{2^{r}} \backslash\{1\}$, there exists a unique $h \in H$ with $a^{h}=b$. Then $b \in K_{2^{r}}^{h} \cap K_{2^{r}}$. Since $K_{2^{r}}$ is TI, then $h \in N_{H}\left(K_{2^{r}}\right)$. Thus $N_{H}\left(K_{2^{r}}\right)$ is regular on $K_{2^{r}} \backslash\{1\}$. Hence it follows that $\left|N_{H}\left(K_{2^{r}}\right)\right|=2^{r}-1$.

(d) Let $r>1$, and let $N_{H}\left(K_{2^{r}}\right)=H_{1}$ be of order $2^{r}-1$. Then $2^{r}-1$ divides $q-1=2^{f}-1$, which happens if and only if $\left|K_{2^{r}}\right|=\left|Z\left(S_{1}\right)\right|$ for the Sylow subgroup $S_{1} \in \operatorname{Syl}_{2}\left(G_{1}\right)$ for a simple Suzuki subgroup $G_{1} \leq G$. This happens if and only if $r \mid f$ and $r>1$.

Suppose that $r>1$ and $\left|H_{1}\right|=2^{r}-1$. This divides $|H|=q-1=2^{2 m+1}-1$. Then $r \mid 2 m+1$, and hence $\left(2^{r}\right)^{k}=2^{2 m+1}$ for some positive integer $k$. Thus if $G_{1}=\operatorname{Sz}\left(2^{r}\right)$, then $S_{1} \in \operatorname{Syl}_{2}\left(G_{1}\right)$ has center $Z\left(S_{1}\right)$ of order $2^{r}$. Conversely, if $2^{r}$ is the size of $Z\left(S_{1}\right)$ for some $S_{1} \in \operatorname{Syl}_{2}\left(G_{1}\right)$ for a simple Suzuki subgroup $G_{1} \leq G$, then $r>1$ and $\left(2^{r}\right)^{k}=2^{2 m+1}$ for some positive integer $k$; hence $2^{r}-1 \mid 2^{2 m+1}-1$.

(e) A non-cyclic elementary abelian 2-subgroup of $G$ is TI if and only if it is the center of a Sylow subgroup $S_{1} \in \operatorname{Syl}_{2}\left(G_{1}\right)$ for some simple Suzuki subgroup $G_{1} \leq G$ or conjugate to it; in particular, Klein four subgroups of $G$ are not TI.

One direction follows from (a); the other direction follows from (b) using (c) and (d).

(f) A non-cyclic elementary abelian 2-subgroup of $G$ is TI if and only if it is of combinatorial depth 3.

If $K_{2^{n}}$ is TI, then it is of combinatorial depth 3 by Remark 2.9. Let $n>1$. If $K_{2^{n}} \leq F$ is not TI, then we have that there exists an element $x_{2} \notin N_{G}\left(K_{2^{n}}\right)$ such that $K_{2^{n}}^{x_{2}} \cap K_{2^{n}} \neq\{1\}$. Let $x_{1} \in F$. Then $x_{1}$ centralizes $K_{2^{n}}$ and we cannot find an element $y$ with $K_{2^{n}} \cap K_{2^{n}}^{x_{1}} \cap K_{2^{n}}^{x_{2}}=K_{2^{n}} \cap K_{2^{n}}^{y}$ such that $y$ also centralizes the intersection since then $y \in F$ and it also centralizes $K_{2^{n}}$. Hence the combinatorial depth of $K_{2^{n}}$ in $G$ is bigger than 3 . 
(8) If $L<F$ is not elementary abelian, then $L$ is not $T I$.

Suppose by contradiction that $L$ is TI and not elementary abelian. Let $I$ be the subgroup generated by the involutions of $L$. If $|I|=2$, then since $F$ does not contain quaternion subgroups, $L \simeq C_{4}$; hence it is not TI by step (6). Let $|I|>2$.

Suppose that $I \cap I^{x} \neq\{1\}$; then $L \cap L^{x} \neq\{1\}$ and $x \in N_{G}(L) \leq N_{G}(I)$. Thus $I$ is also TI; hence it is the center of a Sylow 2-subgroup of a simple Suzuki subgroup of $G$ or conjugate to it. If $x \in N_{G}(I) \backslash N_{G}(L)$, then

$$
I^{x}=I \leq L^{x} \cap L \neq\{1\},
$$

and hence $L$ is not TI. So we may assume that $N_{G}(I)=N_{G}(L)$. Since $I \leq Z(F)$, $F \leq N_{G}(I)=N_{G}(L)$. Thus $L$ is normal in $F$ and $Z(F) L \triangleleft F$. If $x \in L$ with $o(x)=4$, then by the proof of [17, Lemma 5.9, Chapter XI, p. 216], the number of conjugates of $x$ in $F$ is $\left|F: C_{F}(x)\right|=|F| /(2|Z(F)|)=q / 2$. Since $F / Z(F)$ is abelian, all $F$-conjugates of $x$ are in the coset $Z(F) x$; moreover, using that $L \triangleleft F$, we have that they are also in $(Z(F) \cap L) x$. Thus $|Z(F) \cap L| \geq q / 2$. $Z(F) \cap L$ cannot be the center of a Sylow 2-subgroup of a smaller Suzuki subgroup since the order of that subgroup was smaller than $q / 2$. Thus $Z(F) \leq L$. Since $Z(F)$ is normalized by $H$, we have that $L \cap L^{h} \neq\{1\}$ holds for every $h \in H$. Hence $H$ must normalize $L$. However, $H$ acts fixed point freely on $L / Z(F)$. Hence $|L / Z(F)|=q$, and $L=F$. We are done.

Thus we have considered all the possible subgroups up to conjugacy; see Theorem 2.1

Remark 3.1. Let $|Z(F)|=2^{f}$. Not every elementary abelian subgroup of order $2^{n}$, where $1<n \mid f$, is conjugate to the center of a Sylow 2-subgroup of a simple Suzuki subgroup of $G$. The number of subgroups of order $2^{n}$ in $Z(F)$ is

$$
\frac{\left(2^{f}-1\right)\left(2^{f}-2\right) \cdots\left(2^{f}-2^{n-1}\right)}{\left(2^{n}-1\right)\left(2^{n}-2\right) \cdots\left(2^{n}-2^{n-1}\right)} .
$$

If $S_{1} \in \operatorname{Syl}_{2}\left(G_{1}\right)$ for a Suzuki subgroup $G_{1} \leq G$ and $\left|Z\left(S_{1}\right)\right|=2^{n}$, then the number of $H$-conjugates of $Z\left(S_{1}\right)$ is $\left|H: N_{H}\left(Z\left(S_{1}\right)\right)\right|=\frac{\left(2^{f}-1\right)}{\left(2^{n}-1\right)}$, which is strictly smaller than the above number if $n<f$. Thus there are some subgroups, $Z\left(S_{1}\right)$ and its $H$-conjugates, which are TI, and the remaining subgroups of $Z(F)$ of order $2^{n}$ are not TI.

Proof of Corollary 1.6. By part (7) (b) in the proof of Theorem 1.1, we have that $\bigcup_{h \in H} Z\left(F_{1}\right)^{h}=Z(F)$, and the subgroups $Z\left(F_{1}\right)^{h}$ for $h \in H$ intersect trivially. Let $a \in F \backslash Z(F)$ such that $a \in F_{1}$. Since $H$ acts sharply 1 -transitively on the nontrivial elements of $F / Z(F)$, each coset of $Z(F)$ contains an element of a conjugate of $F_{1}$. By the proof of [17, Lemma 5.9, Chapter XI], the element $a$ has $q / 2=\left|F: C_{G}(a)\right|=|F: Z(F)\langle a\rangle|$ conjugates and $a^{-1}$ also. The $F$-conjugates 
of these elements all lie in $Z(F) a$. Thus these give all the elements of $Z(F) a$ since this coset has $q$ elements. Hence each element of $F$ is in a conjugate of $F_{1}$. Since $F$ is a TI set, the conjugating elements are in $N_{G}(F)$.

For the second part, see [14, Proposition 4.13] or the following shorter argument. Suppose that $x \in G \backslash N_{G}\left(F_{1}\right)$ and $a \in F_{1} \cap F_{1}^{x}$ is an element of order 4. Then there exists an element $b \in F_{1}$ such that $b^{x}=a$. By [17, Lemma 11.7, Chapter XI], we have that, in $G$, there are two conjugacy classes of elements of order 4. We know that $K_{G}(a) \neq K_{G}\left(a^{-1}\right)$; otherwise, an element of $H$ would centralize $a^{2}$; similarly, $K_{G}(b) \neq K_{G}\left(b^{-1}\right)$. They are also in different conjugacy classes in $G_{1}$. Thus $a$ and $b$ are conjugate also in $G_{1}$. Hence they are conjugate in $N_{G_{1}}\left(F_{1}\right)$. Thus there exists an element $y \in N_{G}\left(F_{1}\right)$ such that $b^{y}=a$. Hence we have $x y^{-1} \in C_{G}(b)$. Since $x \notin N_{G}\left(F_{1}\right)$ and $y \in N_{G}\left(F_{1}\right)$, then $x y^{-1} \notin N_{G}\left(F_{1}\right)$. However, $x y^{-1} \in C_{G}(b)=Z(F)\langle b\rangle \leq N_{G}\left(F_{1}\right)$. This contradiction shows that there cannot be an order 4 element in two conjugates of $F_{1}$. If $F_{1} \cap F_{1}^{x} \neq\{1\}$, then they have common elements of order 2 ; hence $Z\left(F_{1}\right) \cap Z\left(F_{1}\right)^{x} \neq\{1\}$. We have seen that $Z\left(F_{1}\right)$ is a TI subgroup of $G$; hence $Z\left(F_{1}\right)=Z\left(F_{1}\right)^{x}$. Since $F_{1}$ and $F_{1}^{x}$ do not have common elements of order 4 and all their elements of order 2 are central, hence $F_{1} \cap F_{1}^{x}=Z\left(F_{1}\right)$.

\section{Proof of Theorem 1.3}

Proof of Theorem 1.3. (i) As by [14], except for $N_{G}(F)$, all maximal subgroups have a disjoint conjugate, this holds also for their subgroups; thus any such nontrivial subgroup has ordinary depth 3 .

(ii) This holds since $F$ is TI.

(iii) Now we have to consider the subgroups of $N_{G}(F)=F H$. We want to prove that if the subgroup does not contain $F$, then it has a disjoint conjugate.

Let $U$ be such a subgroup. Let us suppose now that $U=F_{1} K$, where $F_{1}<F$ and $K \leq H$. Then by [24, Theorem 17.3], we have that $U$ is a Frobenius group with kernel $F_{1}=F \cap U$ and complement $K$. Moreover, since $K$ is a characteristic subgroup in the cyclic group $H$, and $H$ is a TI set in $G$, so $K$ is also a TI set in $G$. Let $x \notin F H=N_{G}(F)$. If $U^{x} \cap U=\{1\}$, then we are done.

Otherwise, $U^{x} \cap U \neq\{1\}$. The subgroup $U^{x} \cap U$ cannot contain a nontrivial element $f_{1} \in F_{1}$ since then $f_{1} \in F_{1} \cap F_{1}^{x} \leq F \cap F^{x}$. Since $F$ is TI, we have that $x \in N_{G}(F)$, which is not the case. Then $\left|U^{x} \cap U\right|$ is a divisor of $|K|$. Since $U$ is solvable, by Hall's theorem, $U^{x} \cap U$ is contained in a complement of $F_{1}$, and it can be conjugated in $U$, moreover, in $F_{1}$, to a subgroup of any other complement of $F_{1}$ in $U$. We may suppose that $U^{x} \cap U \leq K \leq H$. Otherwise, let $s \in F_{1}$ be an element such that $\left(U^{x} \cap U\right)^{s} \leq K$. Then $K \geq\left(U^{x} \cap U\right)^{s}=U^{x s} \cap U$ and 
$x s \notin N_{G}(F)$. Thus we can, if necessary, exchange $x$ to $x s$ to get $U^{x} \cap U \leq K$. Let $K_{1}:=U^{x} \cap U$. So we may suppose that $K_{1} \leq K$.

The Frobenius complements of $U^{x}$ are of the form $K^{f_{1} x}$ for some $f_{1} \in F_{1}$. Since $U^{x}=F_{1}^{x} \cup\left(\bigcup_{f_{1} \in F_{1}} K^{f_{1} x}\right)$, for some element $f_{1} \in F_{1}$, we have that

$$
\{1\} \neq K^{f_{1} x} \cap K \leq H^{f_{1} x} \cap H .
$$

Since $H$ is TI, we have that $f_{1} x \in N_{G}(H) \leq N_{G}(K)$, as $K$ is characteristic in the cyclic group $H$. Thus $K^{f_{1} x}=K \leq U^{x} \cap U=K_{1}$. Hence $U^{x} \cap U=K$. Let $l \in N_{F x}\left(F_{1}^{x}\right) \backslash F_{1}^{x}$. Then $\left(F_{1}^{x} K\right)^{l}=F_{1}^{x} K^{l} \leq F^{f_{1} x} K=(F K)^{f_{1} x}$ since $l \in F^{x}$ and $f_{1} x \in N_{G}(K)$.

Now, since $l \in N_{F^{x}}\left(F_{1}^{x}\right)$ and $f_{1} x \in N_{G}(K)$, moreover $\left(F_{1}^{x} K\right)^{l} \leq(F K)^{f_{1} x}$, we have that $F_{1} K \cap\left(F_{1} K\right)^{f_{1} x l}=F_{1} K \cap F_{1}^{x} K^{l} \leq F K \cap(F K)^{f_{1} x}$. The subgroup $F K \cap(F K)^{f_{1} x}$ contains $K$; however, it cannot contain any elements of $F$; otherwise, as $F$ is TI, this would imply that $f_{1} x \in N_{G}(F)$, which is not the case. So $F K \cap(F K)^{f_{1} x}=K$. If $K \cap\left(F_{1}^{x} K^{l}\right) \neq\{1\}$, then since $F_{1}^{x} K^{l}=\left(F_{1}^{x} K\right)^{l}$, for some element $f_{2}^{x} \in F_{1}^{x}$, we have that $K \cap K^{f_{2}^{x} l} \neq\{1\}$. Hence $f_{2}^{x} l \in N_{G}(K)$ since $K$ is a TI set in $G$. However, $l \in F^{x} \backslash F_{1}^{x}$ so $f_{2}^{x} l \in F^{x} \backslash F_{1}^{x}$. Thus a nontrivial element of $F^{x}$ normalizes a Frobenius complement of $F^{x} K$, which cannot happen. Thus $K \cap\left(F_{1}^{x} K^{l}\right)=\{1\}$, and so $F_{1} K \cap\left(F_{1} K\right)^{f_{1} x l}=\{1\}$. Hence $d\left(F_{1} K, G\right)=3$. (If $F_{1}=F$, this proof does not work since then such $l$ does not exist.)

Let us suppose now that $U=F K$, where $K<H$. We want to prove that $d(U, G)=5$. We know, see the proof of [14, Proposition 4.3], that there exist elements $x_{1}, x_{2} \in G$ with $F H \cap(F H)^{x_{1}} \cap(F H)^{x_{2}}=\{1\}$. Hence, by the second part of Theorem 2.6, we have that $d(U, G) \leq 5$. Suppose by contradiction that $d(U, G) \leq 4$. Then, by Definition 2.5 , we have that $m(\chi) \leq 1$ for each irreducible character $\chi \in \operatorname{Irr}(G)$. We will prove that, for $\chi=1_{G}$, this is not true. For let us take a nontrivial irreducible character $\psi \in \operatorname{Irr}(F K / F)$. We will prove that $d\left(\psi, 1_{F K}\right)=2$.

Since $\psi$ can be extended to a nontrivial irreducible character $\theta$ of $N_{G}(F)$ containing $F$ in its kernel, by the proof of [14, Corollary 5.1], $d\left(\theta, 1_{N_{G}(F)}\right)=2$. Hence $d\left(\psi, 1_{F K}\right) \leq 2$.

Suppose by contradiction that this distance was 1 . Then $\left(\psi^{G}, 1_{F K}^{G}\right) \neq 0$. However, $\psi$ can be extended in $|H: K|$ ways to $F H=N_{G}(F)$. Let us suppose that these extensions are $\psi_{1}, \ldots, \psi_{|H: K|}$. These are all the constituents of $\psi^{F H}$. Then

$$
\left(\psi^{G}, 1_{F K}^{G}\right)=\left(\left(\psi^{F H}\right)^{G}, 1_{F K}^{G}\right)=\sum\left(\psi_{i}^{G}, 1_{F K}^{G}\right) .
$$

However, $1_{F K}^{G}=\left(1_{F K}^{F H}\right)^{G}$, and the irreducible constituents of $1_{F K}^{F H}$ are exactly those characters $\phi \in \operatorname{Irr}(F H)$ whose kernel contains $F K$. Thus if $\left(\psi^{G}, 1_{F K}^{G}\right) \neq 0$, then for some $\phi \in \operatorname{Irr}(F H / F K),\left(\psi_{i}^{G}, \phi^{G}\right) \neq 0$. However, by [17, Chapter XI, 
Lemma 5.3], $\left(\phi^{G}, \psi_{i}^{G}\right) \neq 0$ if and only if $\phi=\psi_{i}$ or $\phi=\bar{\psi}_{i}$. We know that $\psi_{i}$ is an extension of a nontrivial irreducible character of $F K / F$; hence neither $\psi_{i}$ nor $\overline{\psi_{i}}$ can contain $K$ in its kernel. Thus $\left(\psi_{i}^{G}, 1_{F K}^{G}\right)=0$ for all $i$ in the above sum; hence $\left(\psi^{G}, 1_{F K}^{G}\right)=0$, and $d(U, G) \not 4$. Thus it is 5 , and we are done.

In [14], we proved that subgroups of type (i) and (ii) have disjoint conjugates. In (iii), we proved above this property for subgroups of type (iii). Conversely, if a non-normal subgroup has a disjoint conjugate, then by the second part of Theorem 2.6, it is of ordinary depth 3 .

\section{Final remarks}

Since for subgroups $L$ of ordinary depth 3 in an arbitrary group $G$, it is not true in general that $L$ has a disjoint conjugate, it is natural to ask the following.

Question 5.1. Let $L \leq G$ be a subgroup of a finite group $G$ of ordinary depth 3 . Is it true that $\operatorname{Core}_{G}(L)=L \cap L^{x_{1}} \cap L^{x_{2}}$ for suitable elements $x_{1}$ and $x_{2}$ ?

We are also interested in a possible converse of Corollary 1.6.

The following group is defined in [16, Example 6.7, Chapter VIII].

Definition 5.2. Let $F=G F\left(2^{n}\right)$, and let $\theta$ be an automorphism of $F$. Let $A(n, \theta)$ be the set of matrices of the form

$$
u(a, b)=\left(\begin{array}{ccc}
1 & 0 & 0 \\
a & 1 & 0 \\
b & a \theta & 1
\end{array}\right)
$$

with $a, b \in F$. Then

$$
\begin{aligned}
u(a, b) u\left(a^{\prime}, b^{\prime}\right) & =u\left(a+a^{\prime}, b+b^{\prime}+a^{\prime}(a \theta)\right), \\
u(a, b)^{-1} & =u(a, b+a(a \theta)) .
\end{aligned}
$$

Then $A(n, \theta)$ is a group of order $2^{2 n}$ with unit element $u(0,0)$.

The following theorem characterizes Suzuki 2-groups; see [16, Theorem 7.9, Chapter VIII].

Theorem 5.3. Let $S$ be a Suzuki 2-group; then

(a) $S^{\prime}=\Phi(S)=Z(S)=\left\{x \in S \mid x^{2}=1\right\}$;

(b) (i) either $S$ is isomorphic to a group $A(n, \theta)$ for some non-identity automorphism $\theta$ of $G F\left(2^{n}\right)$ of odd order and $|S|=|Z(S)|^{2}=2^{2 n}$,

(ii) or $|S|=|Z(S)|^{3}$. 
Remark 5.4. Sylow 2-subgroups of simple Suzuki groups $\mathrm{Sz}(q)$ are isomorphic to certain $A(2 m+1, \theta)$, where $\theta \in \operatorname{Aut}\left(G F\left(2^{2 m+1}\right)\right.$ ) (acting as $x \theta=x^{2^{m+1}}$ ), which is of odd order, thus falls into the category (i) in the previous theorem. However, not every $A(n, \theta)$ is isomorphic to a Sylow 2-subgroup of a simple Suzuki group.

Question 5.5. Let $S$ be a 2-group, and let $H \leq \operatorname{Aut}(S)$. Suppose that $S_{1} \leq S$ is a Suzuki 2-group, which is isomorphic to a Sylow 2-subgroup of a simple Suzuki group $\operatorname{Sz}(q)$. Let $H_{1} \leq \operatorname{Aut}\left(S_{1}\right)$ be a solvable group of automorphisms, acting on the involutions of $S_{1}$ transitively, and let $H_{1} \leq H$. Let us consider the semidirect product $S H$. Suppose further that $S=\bigcup_{x \in S H} S_{1}^{x}$ and $S_{1} \cap S_{1}^{x}$ is either trivial or the common center of them. Is it true that $S$ is a Suzuki 2-group?

Remark 5.6. From Theorem 5.3, it follows that, in Question 5.5, Aut $(S)$ acts transitively on the involutions of $S$, so this conjecture is a weakened form of a conjecture of Gross on finite 2-groups $S$ with more than 1 involution admitting a group of automorphisms that transitively permutes the involutions of $S$, called 2-automorphic 2-groups; see [11]. According to the results of Gross, these groups fall into three classes: (a) homocyclic, (b) of exponent 4 and class 2 of order $|Z(S)|^{2}$ or $|Z(S)|^{3}$ and $S^{\prime}=\mho^{1}(S)=Z(S)$ has exponent 2 , or (c) of exponent 8 and class $3, S^{\prime}$ is homocyclic of order $|Z(S)|^{2}$ and exponent 4 , and $S^{\prime}=\mho^{1}(S)=Z(S)=\left[S, S^{\prime}\right]$ has exponent 2 . The conjecture was that the groups of type (b) are Suzuki 2-groups and those of type (c) do not occur. In the paper [26], it was proved that 2-automorphic 2-groups are of class at most 2, so case (c) does not occur. In the paper [5], it was proved that 2-automorphic 2-groups of type (b) are Suzuki 2-groups if $|Z(S)|^{3}=|S|$. The other case in type (b) is still open. Thus, in Question 5.5, the group $S$ must be of type (b), and in the case $|Z(S)|^{3}=|S|$, the question has a positive answer. In [5], it was also proved that the automorphism group of a Suzuki 2-group is always solvable. In [27], it was proved that, in Suzuki 2-groups $S$ of order $|Z(S)|^{2}$, any two elements of the same order are conjugate in $\operatorname{Aut}(S)$.

Now we summarize the conjectures. Taking into consideration the results of Higman, Shult, Gross, Bryukhanova and Wilkens, the reformulated conjecture is the following: if a finite group $G$ acts on a non-abelian finite 2-group $S$ with more than 1 involution and $G$ is transitive on the involutions of $S$, then $G$ is solvable.

So far, it has been proved that if a finite group $G$ acts on a non-abelian finite 2-group $S$ with more than 1 involution, then if there is a solvable subgroup $G_{1} \leq G$ that acts on the involutions of $S$ transitively, then $G$ is solvable. We ask that if $S$ has some more properties, namely we suppose that $S$ has a special kind of covering of Question 5.5, then $G$ is solvable. 
Acknowledgments. We are grateful for the referee for the helpful comments and suggestions.

\section{Bibliography}

[1] H. Bäärnhielm, Recognising the Suzuki groups in their natural representations, J. Algebra 300 (2006), no. 1, 171-198.

[2] R. Boltje, S. Danz and B. Külshammer, On the depth of subgroups and group algebra extensions, J. Algebra 335 (2011), 258-281.

[3] R. Boltje and B. Külshammer, Group algebra extensions of depth one, Algebra Number Theory 5 (2011), no. 1, 63-73.

[4] J. N. Bray and H. Bäärnhielm, A new method for recognising Suzuki groups, J. Algebra 493 (2018), 483-499.

[5] E. G. Bryukhanova, Automorphism groups of 2-automorphic 2-groups, Algebra Logic 20 (1981), no. 1, 1-12.

[6] S. Burciu, L. Kadison and B. Külshammer, On subgroup depth, Int. Electron. J. Algebra 9 (2011), 133-166.

[7] T. Fritzsche, The depth of subgroups of PSL(2, q), J. Algebra 349 (2012), 217-233.

[8] T. Fritzsche, B. Külshammer and C. Reiche, The depth of Young subgroups of symmetric groups, J. Algebra 381 (2013), 96-109.

[9] F. M. Goodman, P. de la Harpe and V.F. R. Jones, Coxeter Graphs and Towers of Algebras, Math. Sci. Res. Inst. Publ. 14, Springer, New York, 1989.

[10] D. Gorenstein, Finite Groups, Chelsea, New York, 2000.

[11] F. Gross, 2-automorphic 2-groups, J. Algebra 40 (1976), no. 2, 348-353.

[12] A. Hernandez, L. Kadison and M. Szamotulski, Subgroup depth and twisted coefficients, Comm. Algebra 44 (2016), no. 8, 3570-3591.

[13] A. Hernandez, L. Kadison and C. Young, Algebraic quotient modules and subgroup depth, Abh. Math. Semin. Univ. Hambg. 84 (2014), no. 2, 267-283.

[14] L. Héthelyi, E. Horváth and F. Petényi, The depth of subgroups of Suzuki groups, Comm. Algebra 43 (2015), no. 10, 4553-4569.

[15] G. Higman, Suzuki 2-groups, Illinois J. Math. 7 (1963), 79-96.

[16] B. Huppert and N. Blackburn, Finite Groups. II, Springer, Berlin, 1982.

[17] B. Huppert and N. Blackburn, Finite groups. III, Springer, Berlin, 1982.

[18] H. A. Janabi, T. Breuer and E. Horváth, Subgroups of arbitrary large even ordinary depth, Int. J. Group Theory (2020), DOI 10.22108/ijgt.2020.123551.1628. 
[19] L. Kadison, Algebra depth in tensor categories, Bull. Belg. Math. Soc. Simon Stevin 23 (2016), no. 5, 721-752.

[20] L. Kadison and B. Külshammer, Depth two, normality, and a trace ideal condition for Frobenius extensions, Comm. Algebra 34 (2006), no. 9, 3103-3122.

[21] L. Kadison and D. Nikshych, Hopf algebra actions on strongly separable extensions of depth two, Adv. Math. 163 (2001), no. 2, 258-286.

[22] H. Lüneburg, Die Suzukigruppen und ihre Geometrien, Springer, Berlin, 1965.

[23] J. Moori and A. Saeidi, Some designs invariant under the Suzuki groups, Util. Math. 109 (2018), 105-114.

[24] D. Passman, Permutation Groups, W. A. Benjamin, New York, 1968.

[25] M. Suzuki, On a class of doubly transitive groups, Ann. of Math. (2) 75 (1962), 105145.

[26] B. Wilkens, A note on 2-automorphic 2-groups, J. Algebra 184 (1996), no. 1, 199206.

[27] J. P. Zhang, On finite groups all of whose elements of the same order are conjugate in their automorphism groups, J. Algebra 153 (1992), no. 1, 22-36.

[28] The GAP Group, GAP - Groups, Algorithms, and Programming, Version 4.9.1, 2018.

Received March 23, 2020; revised November 24, 2020.

\section{Author information}

Hayder Abbas Janabi, Department of Algebra,

Budapest University of Technology and Economics,

H-1111 Budapest, Múegyetem rkp. 3-9, Hungary;

and Department of Basic Sciences, Faculty of Dentistry,

University of Kufa, Najaf, Iraq.

E-mail: haydera.janabi@uokufa.edu.iq

László Héthelyi, Department of Algebra,

Budapest University of Technology and Economics,

H-1111 Budapest, Múegyetem rkp. 3-9, Hungary.

E-mail: fobaba@t-online.hu

Corresponding author:

Erzsébet Horváth, Department of Algebra,

Budapest University of Technology and Economics,

H-1111 Budapest, Múegyetem rkp. 3-9, Hungary.

E-mail: he@math. bme.hu 